\title{
HF radar QC on azimuth measurement: Dealing with sea echoes seemingly from the land
}

\author{
M.L. Heron; M. O'Shea
}

Echoes that seemingly come from the land occur on both phased array and crossed loop systems. Past practice has generally been to avoid pointing the phased array beam at land targets, and masking out any land echoes from the radials data file for crossed loop system. In an attempt to avoid this sanitising of data this paper addresses possible causes and evidence-based procedures for dealing with the effect. Phased array radars do get echoes when the beam is pointed towards land and it is shown that the echo power drops significantly when the main beam is swept across the coast from sea to land. This change in amplitude can be used to develop a robust criterion for the elimination of land echoes. For crossed loop direction finding systems the error in determination of the bearing of sea echoes is used to identify a coastal land area in which the echoes are actually from true sea scatter from the adjacent ocean. It is suggested that these echoes should be included in the estimation of sea surface currents. 